\title{
CONVERGENCIAS / DIVERGENCIAS DE COMUNIDADES LINGÜÍSTICAS EN EL MARCO ESPAÑOL*
}

Georg Kremnitz

Universität Wien

* Agradezco a Helga Lion su lectura atenta y crítica de mi texto. Todos los errores que quedan son de mi responsabilidad. 

El tema de la intervención que me han propuesto - y agradezco mucho a los organizadores que me hayan obligado nuevamente a reflexionar sobre estos problemas- es tan complejo que necesita algunas consideraciones previas, antes de comenzar con el debate. Por consiguiente, evocaré, en primer lugar, algunos elementos de las posiciones básicas que defiendo; en segundo lugar, hablaré sobre el marco constitucional y legal de toda política lingüística en el Estado español actual, sobre algunos antecedentes históricos y normativos — que no son los mismos para todas las lenguas de España-; en tercer lugar, sobre algunos aspectos de la demografía en general y de la demografía lingüística en particular. Habrá que añadir algunas observaciones sobre hechos nuevos que la Constitución de 1978 no pudo tener en cuenta. Y, finalmente, expondré las convergencias y divergencias de las políticas lingüísticas en el Estado y las consecuencias que tienen sobre las diferentes comunidades lingüísticas.

Por lo pronto, permítanme unas pocas frases para definir el tema central de hoy: sabemos todos que el fin de la dictadura franquista tenía y tiene una importancia particular no solamente para España, sino también para toda Europa. No fue solamente la implosión, con pocas víctimas (aproximadamente 400 muertos, si mis informaciones son correctas), de una de las dictaduras más inflexibles, sino que también fue la primera vez que el centralismo político, herencia de la revolución francesa en muchos estados, fue restringido en un grado muy avanzado con la (en parte no programada ni prevista) creación del Estado de las Autonomías y el reconocimiento — por lo menos parcial— del multilingüismo y de la pluralidad cultural de este estado. Más adelante, fue considerado como uno de los precursores de los acontecimientos y, sobre todo, de las soluciones de política cultural en Europa oriental. Hay observadores que consideran que, si la transición se hubiese producido dos lustros más tarde, en el momento de la creación de los nuevos estados en Europa (en algunos casos solamente la recreación de estados antiguos), la integridad del Estado español no hubiera sido garanti- 
zada. Este es todavía uno de los traumas (no admitidos) de una parte de los nacionalistas españoles, y por eso constituye un freno considerable para una política lingüística verdaderamente pluralista; hoy en día hay estados que han superado al Estado español en este sector a pesar de que, en aquel entonces, haya sido el ejemplo que había que imitar. Y eso me parece también una de las razones más importantes de la amargura — de ambos lados - que prevalece siempre en las discusiones sobre los aspectos de los derechos lingüísticos en España. Las discusiones a menudo carecen de serenidad y de capacidad de intercambio de perspectivas; esta última se exigiría sobre todo por parte de los defensores del lado estatal porque aún continúa teniendo muchos más recursos que los defensores de las periferias.

\section{ALGUNAS POSICIONES CIENTÍFICAS BÁSICAS DE UNA INTERPRETACIÓN COMUNICATIVA DE LOS HECHOS DEL LENGUAJE}

Ferdinand de Saussure distinguió entre dos aspectos del signo lingüístico — la imagen acústica y el concepto- que son indisociables uno de otro como las dos caras de una misma moneda. Las ciencias de la comunicación poseen un término comparable: cada vez que nos encontramos en una situación de comunicación activamos una tensión entre una función comunicativa y una función demarcativa (o identitaria) de nuestro discurso. Hablamos para comunicarnos con los demás, pero al mismo tiempo, como consecuencia de las particularidades de nuestra manera de hablar, nos demarcamos de los demás. Los diferentes círculos concéntricos de facilidad de comunicación significan también los mismos grados de la identidad colectiva. En la comunicación cotidiana estas particularidades no tienen mucha importancia. Pero cuando nos encontramos frente a interlocutores inhabituales se nota cada vez más. La demarcación no es únicamente espacial, también puede tratarse de aspectos sociales, sexuales, etc. Se nota en rasgos colectivos pero también a nivel individual y puede concernir hábitos fonéticos, el vocabulario, la gramática —-finalmente todos los elementos de la comunicación. Podríamos hablar de círculos de comunicación. Los hablantes tienen una praxis de distinción y de interpretación que se formó paralelamente a la capacidad de hablar, la cual se ejerce cotidianamente. Hay que 
insistir sobre el hecho de que la recepción lingüística no es un hecho pasivo y mecanicista, sino un esfuerzo muy complejo de interpretación de cadenas sonoras. Se podría hablar de un trabajo de comprensión. Si las cadenas sonoras que llegan a nuestros oídos son muy diferentes de las que interpretamos normalmente, podemos llegar a un punto límite a partir del cual nuestros esfuerzos dan resultados insuficientes o nulos. En este mismo punto, la demarcación llega a ser más fuerte que la comunicación: nos encontramos en los límites de nuestro potencial comunicativo. Por cierto, los límites pueden ser influidos hasta un cierto grado por nuestra salud actual, por la atención que prestamos a nuestro interlocutor o por nuestra competencia (o incompetencia) en un dominio particular, por ejemplo. Lo que se puede afirmar sobre la comunicación oral, vale también — con matizaciones - para todas las otras formas y todos los medios de comunicación $(2003 ; 2008)$.

Naturalmente, estos círculos de comunicación no son invariables. Acontecimientos o accidentes históricos pueden crear obstáculos para las relaciones de comunicación habituales, o, al contrario, agrandar los círculos de comunicación ya existentes. Por ejemplo, la creación de las lenguas «nacionales» en Europa (y más adelante en otras partes de nuestra tierra) se produjo por un doble movimiento de engrandecimiento de los círculos (locales y regionales) de comunicación por un lado y, a la vez, de creación de fronteras comunicativas entre estados vecinos por otro lado (cf. Kremnitz, 2009). Actualmente observamos un aumento mundial del círculo de comunicación del inglés, tanto en el sentido diatópico como en los sentidos diastrático y diafásico. Al mismo tiempo, se acentúa un movimiento contrario que pretende cultivar variedades lingüísticas pequeñas con pocos hablantes para conservar rasgos identitarios y demarcativos menos abstractos. Pero como las necesidades de comunicación aumentan con la movilidad creciente, muchas veces los hablantes que usan estas «pequeñas» lenguas para demarcarse claramente de otros y demostrar su identidad utilizan -necesariamente- para la comunicación tanto a distancia como cotidiana lenguas de contacto — que suelen ser las grandes lenguas dominantes con muchos hablantes.

En los límites de los círculos comunicativos, lenguas diferentes (o mejor dicho: sistemas de comunicación interpretados como lenguas diferentes) entran necesariamente en contacto. Esta coexistencia se debe al intercambio de objetos 
y a la necesidad de comunicación práctica. Por eso, las lenguas se influyen mutuamente. Estas influencias se pueden dar por la mera práctica comunicativa, pero la creación de los estados modernos conllevó una ideologización creciente de las lenguas. Esta se hizo, sobre todo, en dos momentos históricos: durante el Renacimiento, que reemplazó el dominio del latín por el de las lenguas de poder de aquel entonces, y, sobre todo, con la aparición del nacionalismo moderno, en relación con y como consecuencia de la revolución francesa. El nacionalismo moderno pretende que entre nación y lengua haya un vínculo necesario (lo que se refuta fácilmente con experiencias históricas), además insiste en el monolingüismo natural del hombre (lo que también no es correcto: el hombre tiene capacidades multilingües, como sabemos todos). Al mismo tiempo se intensifican los esfuerzos de política lingüística (que empezaron ya en la fase del Renacimiento) con la clara voluntad de crear estados monolingües, aunque casi ninguna frontera política en Europa haya, hasta hoy, observado este criterio. Del contacto lingüístico natural se llega de esta manera al conflicto lingüistico que opone una lengua dominante a una (o varias) lenguas dominadas. Queda claro, por tanto, que las instituciones estatales están al servicio de la propagación de la lengua dominante e intentan minimizar el empleo de la(s) lengua(s) dominada(s). Si los hablantes de la lengua dominada consideran su praxis lingüística como parte de su identidad colectiva (ya sea por razones de nacionalismo, ya sea por otras), no aceptarán las tendencias asimiladoras de la política estatal, por lo que nos encontramos ante un conflicto lingüístico agudo. Si la política estatal es menos opresiva o si ambos grupos reconocen que el hombre tiene genéticamente un potencial plurilingüe, existe simplemente un potencial conflictivo que, por un cambio ideológico o político por parte de solo uno de los grupos, se puede transformar en conflicto agudo. En un pasado no muy lejano, hemos observado más de un desarrollo de este tipo. Pero, en general, en estos últimos decenios el potencial conflictivo en casi todos los países de Europa ha disminuido considerablemente por actos de reconocimiento de las minorías autóctonas (a su vez, hay que decirlo claramente, por una asimilación parcial o casi completa de muchas minorías, y, por eso, de una disminución del "peligro» que pueden encarnar en los ojos de los defensores ciegos del Estado). La situación es un poco diferente en lo que se refiere a las minorías de inmigración reciente (cf. Kremnitz, 2003). 
En los estados modernos, la organización política de las relaciones comunicativas es en general compleja y no carece de contradicciones internas. Se puede distinguir entre una política lingüística «explícita», que pretende reglamentar de manera abierta los problemas de la comunicación, y una política «implícita», que se ocupa de otros dominios que pueden tener consecuencias para la comunicación. Esta diferenciación se puede aplicar a dos términos propuestos, hace más de cuarenta años, por la sociolingüística catalana y generalizados desde entonces, a saber, el de estatus y el de prestigio. El término de estatus se refiere a la situación legal de una lengua, mientras que el prestigio equivale a la reputación de la lengua, ya sea en el interior de su grupo de hablantes ("prestigio interno»), o bien en la parte de hablantes de otras lenguas ("prestigio externo»). Normalmente, el estatus es un elemento importante dentro de la política lingüística explícita, mientras que el prestigio forma parte de la política lingüística implícita (pero puede tener consecuencias explícitas: el prestigio exterior del inglés en la actualidad provoca su integración en los currículos de las instituciones educativas). En general, a un estatus elevado le corresponde un prestigio (al menos interno) alto; en caso contrario, eso indicaría la existencia de un conflicto relativamente agudo (cf. Kremnitz, 2003).

Entre estos dos términos aparece otro, que es el valor comunicativo de las lenguas. Por este término, Roberto Bein y yo intentamos hacer visible que entre estatus y prestigio hay un tercer elemento, difícil de definir, que es la praxis efectiva de un idioma en un territorio / espacio definido en un momento histórico determinado. Lo que importa es que al mismo tiempo puede ser muy diferente al estatus y al prestigio. Permítanme darles un ejemplo: en nuestros días, las lenguas de los recién inmigrados en Europa en general no gozan de ningún tipo de reconocimiento legal y tienen un prestigio exterior bajo (lo que puede incidir rápidamente sobre el prestigio interior), porque los hablantes pertenecen mayoritariamente a los grupos más modestos de la sociedad de origen — como lo serán de la sociedad de acogida. Pero estas lenguas tendrían un valor comunicativo bastante elevado porque las encontramos en situaciones y contextos múltiples; si los hablantes de las lenguas autóctonas tuviesen más capacidades en estas lenguas, no solo se podría mejorar la comunicación global, sino que también la cohesión interna de nuestras sociedades y, quizás, hasta el interés de los inmigrados de aprender las lenguas autóctonas podría acentuarse (cf. Bein, 2001; Kremnitz, 2003). 
Quedémonos con estas pocas reflexiones generales y pasemos al problema en concreto.

\section{EL MARCO CONSTITUCIONAL Y LEGAL: LA CONSTITUCIÓN Y LAS AUTONOMÍAS}

Todos sabemos que todo comienza con la Constitución Española de 1978. Con ella se organizó la segunda parte de la transición política e institucional, se evitaron, en lo posible, los errores de la Constitución de 1931 y, a la vez, se mantuvo el equilibrio entre las fuerzas democráticas en progreso y los representantes de la dictadura franquista que aún tenían en aquel momento una influencia política y social considerable. Por estos motivos el texto de la Constitución es un compromiso y hasta contiene ciertas contradicciones internas. En realidad, no contiene mucho sobre los derechos lingüísticos, pero hay que comprender que algunos artículos que aclaran otros problemas tienen consecuencias lingüísticas de forma indirecta. El artículo clave es, como todos sabemos, el artículo 3, que crea un sistema de tres / cuatro niveles diferentes de lenguaje: el castellano como lengua del Estado en la cima, las lenguas cooficiales en las respectivas Comunidades Autónomas, las «modalidades lingüísticas de España» y todas las demás lenguas que se hablan en el territorio español que no entran en una de las categorías anteriores (y que, en consecuencia, se podrían considerar, desde el punto de vista de este texto, como no lenguas). Además, el art. 3.1 dice que «todos los españoles tienen el deber de conocerla y el derecho a usarla». Con esta frase se establece una diferencia enorme entre el castellano y todas las otras lenguas (y no lenguas). Desde la opinión pública que tradicional y mayoritariamente piensa que el hombre es por naturaleza monolingüe, esta frase implica que el plurilingüismo autonómico es a lo mejor un rasgo identitario (destinado a desaparecer en un futuro). Según esta concepción, hay que aceptarlo provisionalmente, pero es una mera concesión otorgada a las periferias. No tiene verdadera importancia comunicativa porque la Constitución prevé que todos deben saber el castellano.

En el art. 20.3 se reconoce el derecho al acceso a los medios de comunicación, en el art. 148.1.17 se estipulan los derechos de las comunidades autóno- 
mas en la enseñanza de las respectivas lenguas autónomas y en la disposición final se prevé la publicación de la Constitución «también en las demás lenguas de España». Pero hay (es verdad: pocos) otros artículos que se podrían calificar de política lingüística implícita. Personalmente, dos me parecen importantes: el art. 2, que fija «la indisoluble unidad de la Nación española, patria común e indivisible de todos los españoles» y que crea, al mismo tiempo, la diferencia entre esta «Nación española» y las «nacionalidades y regiones que la integran». Eso quiere decir claramente que no hay posibilidad de reclamar, según esta Constitución, la independencia política de cualquier parte del Estado español. Se puede argumentar que la mayoría de las constituciones en el mundo conocen fórmulas comparables (una de las raras excepciones fue la Unión Soviética), pero en el caso de tensiones políticas o lingüísticas hay ciertas soluciones que no se pueden discutir de manera abierta sin que los que lo hacen caigan en seguida en la trampa de la anti-constitucionalidad. Pero dice también, al menos implícitamente, que el Estado no tiene confianza suficiente en su propia atracción como modelo de organización. Por vía democrática, un cambio en este dominio no es posible; si la voluntad de los ciudadanos de una parte del Estado quiere desprenderse de este conjunto, solamente es posible a través de la ruptura de la Constitución actual. Finalmente, instituye una diferencia de clase entre la «Nación» y las «nacionalidades» (conocida, por ejemplo, en la Constitución Soviética de 1937 y elaborada por pensadores de izquierdas al principio del siglo XX para solucionar la tensión entre solidaridad de clase y cohesión nacional, cf. Bauer, 1907/1924; Stalin, 1913). El art. 145.1 estipula que «en ningún caso se admitirá la federación de Comunidades Autónomas»; esta frase es también una limitación fuerte a una (posible) voluntad democrática. Por eso se relativiza con la cuarta disposición transitoria que prevé una posible integración de Navarra en la CAV / Euskadi.

Los estatutos de autonomía de la primera generación, productos directos de esta Constitución, se pueden clasificar en tres grupos: los que reconocen una lengua como cooficial (Euskadi, Catalunya, Galicia, Comunidad Valenciana, parcialmente Navarra, Islas Baleares), los que prevén proteger sus modalidades (Asturias, Aragón) y los que no tienen ninguna referencia lingüística, que son la mayoría. Como estos primeros estatutos de autonomía son generalmente bastante breves, los artículos dedicados a las lenguas cooficiales son también lacó- 
nicos: todos (salvo Navarra y la Comunidad Valenciana' ${ }^{1}$ declaran la lengua cooficial «lengua propia»y retoman de esta manera una figura argumentativa que ya aparece en las discusiones del siglo XIX tardío, sobre todo en Catalunya ${ }^{2}$. Implícitamente, esta formulación podría indicar que la lengua autóctona tendría que ocupar el primer lugar en el mercado lingüístico autonómico, pero que por razones políticas no es posible en el momento de la redacción de los estatutos. Todos insisten en la igualdad de derechos de ambas lenguas, aunque con matizaciones diferentes. En Catalunya se estipula, art. 3: «La lengua propia de Cataluña es el catalán. El idioma catalán es el oficial de Cataluña, así como lo es también el castellano, oficial en todo el Estado español». Esta fórmula indica una presencia social fuerte y reconoce, casi de mala gana, la superioridad legal del castellano. El estatuto de Euskadi prevé, art. 6: «El euskera, lengua propia del Pueblo Vasco, tendrá, como el castellano, carácter de lengua oficial en Euskadi». Esta formulación indica que la situación de igualdad todavía no existe (se podría discutir sobre el valor semántico de la forma verbal con relación al futuro) y que hay que prepararla (la elaboración del batua todavía no estaba terminada en el momento de la redacción del texto). Además de las precisiones ya citadas en relación con la promoción del gallego, el estatuto de Galicia de 1981 insiste, en el art. 5.3: «Los poderes públicos de Galicia [...] potenciarán la utilización del gallego en todos los órdenes de la vida pública, cultural e informativa [...]». Las regiones con lengua cooficial dejan los detalles de la reglamentación a leyes autonómicas (que alguna vez se hacen esperar).

Actualmente estamos en un período en el cual algunas de las regiones con lengua cooficial ya han aprobado un nuevo estatuto. Sobre todo en Catalunya este segundo estatuto de 2006 difiere notablemente del primero. Es mucho más amplio (223 artículos, más algunas disposiciones adicionales, etc. en comparación con 57 artículos, más algunos aditivos en la primera versión) y es mucho mas explícito. Además, el Parlamento de Catalunya declaraba, con una amplia mayoría, Catalunya como «nación», denominación que las Cortes españolas no aceptaron, limitaban las aspiraciones catalanas a las de mera «nacionalidad» (art.

\footnotetext{
${ }^{1}$ La Comunidad Valenciana lo hará en la segunda versión del estatuto, de 2006.

${ }^{2}$ La Dra. Ursula Wurl, de la Universidad de Viena, está preparando un trabajo que tendrá que establecer la historia de este concepto. Cf. también la contribución de Juan Carlos Moreno Cabrera en este libro, pp. 83-125.
} 
1) y rechazando la autodefinición de los catalanes como «nación» en el preámbulo (que no tiene valor jurídico). En junio de 2010, el Tribunal Constitucional de España anuló algunas disposiciones del estatuto de 2006 e interpretó otras; las consecuencias de esta sentencia todavía no son claras (cf. art. 6). En lo que se refiere al derecho lingüístico, este nuevo estatuto proclama, en el art. 6.1, el catalán como lengua propia de Catalunya y reutiliza este viejo argumento. En el art. 6.2 se declara que «el català és la llengua oficial de Catalunya. També ho és el castellà, que és la llengua oficial de l'Estat espanyol». Estas fórmulas dan claramente el primer lugar al catalán y relegan el castellano al segundo lugar. Pero el párrafo continúa, en el mismo apartado: «Totes les persones tenen el dret d'utilitzar les dues llengües oficials i els ciutadans de Catalunya tenen el dret i el deure de conèixer-les». Con este apartado se relativiza el art. 3.1 de la Constitución Española, exigiendo de un grupo de ciudadanos conocer otro idioma distinto del castellano - al lado de este- y reforzando, de esta manera, la denominación de lengua propia. Por otro lado, la creación de una categoría "ciudadanos de Catalunya» hará necesaria, algún día, una definición de esta especie de seres humanos. Y estamos en una contradicción bastante abierta entre una y otra voluntad democrática, la de Catalunya y la de España. Hay que añadir que las limitaciones efectuadas por las Cortes españolas muestran claramente que la voluntad democrática española tiene más peso que la catalana, es decir, que los directamente afectados tienen menos posibilidades de expresar su opinión que otros que no lo son. Parece difícil resolver esta contradicción mediante un compromiso que respete las posiciones de ambos lados y que encaje en el marco de la Constitución vigente. A pesar de estos artículos, que dan la impresión de progresos del lado catalanista, personalmente considero que el nuevo estatuto es un texto que trata de reducir los efectos de los inconvenientes: el hecho de estipular con tantos detalles los derechos y las obligaciones de cada lado, no solo en el dominio lingüístico, implica que ambos lados no han logrado, en más de cinco lustros de vigencia del primer estatuto, crear un clima de confianza mutua que acepte abiertamente la existencia y los intereses del lado contrario. En una situación asimétrica como la que crea la Constitución de 1978, estaría claro que la parte dominante, es decir, el Estado español, hubiera tenido que inspirar confianza al elemento más débil, el autonómico, para convencerlo de su disposición a no solamente cumplir con la letra, sino también con 
el espíritu de la Constitución, concediéndole, alguna vez, más de lo mínimo legal. Esta confianza mutua no se ha creado en las autonomías en las que existe una tradición política propia. Hay que constatar que, sobre todo en los primeros años de democracia - particularmente importantes para crear una atmósfera armoniosa-, los representantes de los gobiernos centrales sucesivos han descuidado completamente este aspecto de su comportamiento. El precio político y atmosférico de tal negligencia es muy alto. Pero los detalles del nuevo texto se explican también por la necesidad, sentida por el Parlamento de Catalunya, de proteger espacios comunicativos del catalán que algún día podrían ser reivindicados por defensores del castellano o por el poder central ${ }^{3}$. Para decirlo claramente: en mi opinión es un texto sobre todo defensivo y poco ofensivo. Las otras reformulaciones de estatutos han sido menos conflictivas, salvo que en Euskadi no haya sido posible lograr una solución que produjese un nuevo texto legal.

Lo que se pudo observar durante estos tres decenios desde la nueva Constitución es que algunas regiones, que hasta ahora se limitaron a reconocer «modalidades lingüísticas», comienzan a reorientar sus políticas en dirección a un reconocimiento más elevado de estas. El caso más explícito es el de Asturias, donde el asturiano hoy goza de una situación que se podría calificar como intermedia, pero hay una política lingüística que en muchos dominios se inspira en las políticas autonómicas de cooficialidad. Naturalmente, hay un problema de número de hablantes (los que hablan asturiano con alguna regularidad son menos que los que hablan euskera), por otro lado el asturiano no se habla únicamente en Asturias, mientras que el problema se complica por la presencia del gallego en esa comunidad. Estos problemas prácticos (o considerados como tales) impiden hasta la fecha un consenso político suficientemente amplio para llegar a una reevaluación legal del asturiano. El caso de Aragón también es complicado, porque al lado del aragonés existe la franja catalana, y ambos grupos - los que hablan catalán y los que hablan aragonés - son muy minoritarios. Naturalmente, las primeras razones para que tales movimientos se intensifiquen son

${ }^{3}$ Se podría interpretar la política actual de Catalunya de cooficializar el occitano aranés en toda Catalunya, a pesar de las relaciones históricas y actuales entre los Países Catalanes y las tierras occitanas, como elemento implícito de la misma política: exigimos del Estado español un comportamiento comparable al que estamos desarrollando para nuestra propia minoría. 
identitarias, pero también hay una emulación entre autonomías, que ven que en conjunto es «interesante», desde un punto de vista legal, pero también financiero, disponer de una lengua cooficial. Estas reflexiones pueden producir consecuencias, hasta la fecha menores, también sobre autonomías como Andalucía e, incluso, Extremadura. Pero los grupos que defienden estas diferencias regionales no tienen tradiciones nacionalistas comparables a las anteriores.

\section{ANTECEDENTES HISTÓRICOS Y NORMATIVOS}

Las historias de los renacimientos lingüísticos y culturales de los Países Catalanes, de Euskal Herria y de Galicia son suficientemente conocidas para que me limite aquí a unas pocas evocaciones. El primero de estos movimientos que aparece en la historia es la Renaixença catalana, que se hace visible con la publicación del poema $A$ la Pàtria. Trobes de Bonaventura Carles Aribau en 1833, después sigue el Rexurdimento en Galicia en 1863 con la publicación de los Cantares gallegos de Rosalía de Castro, y finalmente el Eusko Pizkundea, a finales del siglo XIX. Mientras que los dos primeros siguen los esquemas propuestos por Miroslav Hroch — según él, un nacionalismo incipiente empieza por obras literarias y la recuperación de la historia del grupo, después siguen primeros conceptos políticos elaborados en círculos restringidos y solamente en un tercer paso encontramos la adhesión de un público amplio-, en el caso de los vascos todo se acelera rápidamente, de manera que podemos decir que lo cultural y lo político se presentan casi al mismo tiempo (cf. Hroch, 1968; 2005). Conocemos la importancia que tuvo, finalmente, el fracaso de la Primera República española, sobre todo para los catalanes, y después la derrota de la guerra del 98. Sabemos que en este momento la politización del movimiento renacentista en Catalunya hace progresos enormes, mientras que en las demás partes de los Países Catalanes el movimiento se mantiene (sobre todo) a nivel cultural. Paralelamente, la politización del autonomismo gallego fue escasa, mientras que el movimiento vasco tiene inmediatamente un éxito público considerable.

En cada uno de los casos, la lengua del grupo adquiere una importancia creciente. En el caso del catalán esta importancia aboca, después de casi medio siglo de discusiones, a la creación de una forma referencial moderna, la de Pompeu Fabra, que 
tiene un amplio éxito público en muy poco tiempo, y a pesar de la oposición de algunos grupos interiores del Principat, pero sobre todo en las demás partes del dominio lingüístico. Este éxito permite a la Generalitat de 1931 empezar con una política lingüística de cooficialdad, a pesar de los retoques al estatuto de autonomía finalmente aceptado por las Cortes españolas en 1932. Las normas fabrianas se aceptan al final también bastante bien en las demás partes del dominio. Sin el comienzo de la Guerra Civil en 1936, una evolución en dirección a un uso de la lengua en todos los dominios de la comunicación hubiera sido posible. Para los otros dos grupos los progresos son más lentos. Si bien en 1919 se funda Euskaltzaindia, la Academia Vasca, la elaboración de una normativa no se empieza inmediatamente, a pesar de las preocupaciones normativas de su primer presidente, Resurrección María de Azkue. Será solamente durante la reunión de Arantzazu, en 1968, cuando se decide la elaboración del batua y Koldo Mitxelena toma la dirección de las operaciones. Se sabe que en el momento de la creación de la autonomía, en 1979/80, este trabajo aún no estaba terminado, lo que obligaba a las instituciones autonómicas a empezar con su trabajo de normalización institucional con fundamentos no del todo estables. Hoy día este trabajo está concluido y me parece que la lengua referencial — después de algunas oposiciones que se producen siempre en tal situación porque, por un lado, hay gente que defiende concepciones normativas diferentes $y$, por otro lado, los usuarios no aceptan tan fácilmente tales innovaciones, que significan nada menos que un cambio de las condiciones comunicativas de una lengua (por eso el argumento que conocemos todos: la nueva lengua referencial no es la que hablamos, argumento que a la vez es relativamente correcto, pero sin gran importancia) - encuentra una aceptación cada día más grande. Naturalmente, el aumento de la producción escrita y mediática por algo está en este proceso. El caso es más complicado en lo que se refiere al gallego, porque en el momento de la autonomización todavía no existía una forma referencial elaborada. Esta fue elaborada bastante rápido. En 1982 una comisión conjunta de la Real Academia Galega y del Instituto da Lingua Galega propuso la norma actual (con pequeños retoques en 1995 y 2003), aceptada por la Xunta de Galicia, pero hasta la fecha el consenso no es total: una parte de los defensores del gallego pensaba y, continúa pensando, que una orientación más decidida hacia la lusofonía hubiera limitado las influencias del castellano (y del centro del Estado), al mismo tiempo que potenciaría el valor comunicativo de la lengua y su fuerza simbólica. Aquí no es momento para discutir 
este problema —es suficiente recordar que cada elaboración normativa contiene un cierto grado de voluntarismo- pero el resultado es que este conflicto continúa influyendo negativamente en el proceso de normalización y también en el prestigio de la lengua. Todas estas contradicciones repercuten sobre el valor comunicativo y los resultados de la política lingüística. También en Asturias el problema de la forma referencial sigue siendo importante.

La existencia de una muy fuerte conciencia colectiva tanto catalana como vasca se prolonga en la formación de los primeros gobiernos autonómicos: en ambos casos los partidos no estatales obtienen mayorías relativas o absolutas en los parlamentos, y por eso las políticas de afirmación autonómica se acentúan, dando ambas autonomías una importancia clave a las lenguas autóctonas. Es evidente que el punto de partida es muy diferente. En Catalunya, la inmensa mayoría de los habitantes entiende y habla el catalán, mientras que en Euskadi los euskaldunes solamente forman una minoría. Por eso las actuaciones prácticas tienen que diferenciarse considerablemente, pero el espíritu, en definitiva, es el mismo. Se podría cualificar por la insistencia en el concepto de lengua propia que se utiliza en estas dos autonomías. En las otras regiones con lenguas autóctonas los gobiernos le dan menos importancia a este concepto. Lo que conlleva que los esfuerzos reales para promocionar las relativas lenguas autóctonas son mucho menores. Este es el caso de Galicia, en donde durante bastante tiempo, afirmaciones rotundas por parte de los gobiernos sucesivos no iban acompañadas de una política coherente. Muchas veces la política se señalaba por gestos simbólicos, pero no consideraba suficientemente el problema del prestigio de la lengua autóctona y una institucionalización clara de la política en su favor. En los casos de las Islas Baleares o de Navarra, se observan comportamientos comparables. Pero los cambios de mayoría en las Islas, por ejemplo, muestran que ciertas alternativas sí son posibles. El caso más espectacular es el de la Comunidad Valenciana, que, después de comienzos prometedores cae, a partir del momento en el que el PP gana las elecciones en 1994, en una política de secesionismo lingüístico, que tiene como resultado la afirmación del papel del castellano, y el claro incumplimiento de la constitución y de los estatutos de autonomía; estos prevén un bilingüismo oficial simétrico que está lejos de alcanzarse. Es evidente que una voluntad política de diferenciación no es lo suficientemente fuerte para tener un papel más importante, pero es interesante observar tam- 
bién la instrumentalización de la política cultural. Visto desde afuera, al observador le llaman la atención los esfuerzos de autolimitación que se llevan a cabo.

Actualmente nos encontramos en una nueva situación que empieza con la fecha de 2003: todos los gobiernos autonómicos están, por lo menos en parte, en manos de partidos estatales. El presidente de la Generalitat Catalana pertenece al PSC / PSOE, al igual que el lehendakari de Euskadi; el presidente de la Xunta de Galicia es miembro del PP, como también el presidente de la Generalitat Valenciana, etc. El único presidente regional que no es de un partido estatal, que yo sepa, es el de las Islas Canarias y hasta la fecha no ha formulado reivindicaciones lingüísticas. Es verdad que en los gobiernos de Catalunya y de las Islas Baleares algunos partidos no estatales desempeñan un rol importante, pero en este último decenio la reputación internacional de los gobiernos autonómicos ha empeorado. Jordi Pujol fue mundialmente conocido, su sucesor actual lo es únicamente por especialistas - y eso no se debe solamente a la personalidad de Pujol. El caso más delicado me parece que es el de Euskadi, donde todos los partidos abertzales fueron, desde la elecciones de 2009, por primera vez excluidos de la participación gubernamental; las consecuencias de esta unión estatal contra el conjunto de los grupos autonomistas en Euskadi todavía no son previsibles.

En consecuencia, se pone de relieve que, por primera vez desde la proclamación de la Constitución de 1978, hay un conjunto de fuerzas políticas estatales que intentan frenar y hasta tratan de interrumpir el proceso empezado en aquel entonces. Es evidente que los grupos autonómicos lograron mucho pero, por otro lado, aún quedan tareas sumamente importantes por realizar.

\section{EL MARCO DEMOGRÁFICO Y DEMOLINGÜÍSTICO}

Un elemento importante para entender las evoluciones lingüísticas es el cambio demográfico. Quiero indicar algunos datos a continuación:

La población de España aumentó entre 1975 y 2010 de la manera siguiente:

\begin{tabular}{ll} 
Habitantes en 1975: & 35470000 (Fischer Weltalmanach, 1977) \\
\hline Habitantes en 1981: & 37583000 (Ferrer, 1985) \\
\hline Habitantes en 2010: & 46030000 (www.ine.es, 2010) \\
\hline
\end{tabular}


En tres de las diferentes regiones autonómicas, la evolución es la siguiente (según el INE [www.ine.es, 2010]; los porcentajes se refieren al total de España):

\begin{tabular}{lll} 
& 1981 & $\mathbf{2 0 1 0}$ \\
\hline Catalunya & $5956414(15,85 \%)$ & $7298385(15,85 \%)$ \\
\hline Euskadi & $2141809(5,70 \%)$ & $2139294(4,65 \%)$ \\
\hline Galicia & $2811912(7,48 \%)$ & $2738254(5,95 \%)$ \\
\hline
\end{tabular}

Está claro que se trata de consideraciones que pueden proporcionar solamente una impresión aproximativa. Pero, según estas cifras, solo Catalunya pudo mantener su peso demográfico, mientras que las otras dos comunidades han perdido importancia demográfica. Observaciones similares se podrían hacer si comparamos las cifras de las otras comunidades autónomas con una situación lingüística especial. Pero concentrémonos en estos datos, de momento.

Si observamos ahora la evolución de las competencias lingüísticas, vemos que las incongruencias continúan. Hay que decir que las cifras, oficiales o semi-oficiales, que tengo a mi disposición no siguen siempre los mismos criterios. Por eso tengo que comparar un poco manzanas con peras, como se suele decir en alemán. Pero son suficientemente explícitas para darnos una impresión.

Los resultados estadísticos de las competencias están muy detallados para Catalunya. Se puede observar un progreso claro en todos los dominios. Si para 1981 dispongo solo de una indicación aproximativa de los que entienden el catalán: 80,5\% de la población de más de 2 años (Querol i Puig, 2006, p. 73), las indicaciones ulteriores son mucho más precisas. Del descuento de la población de dos años y más resultan las siguientes:

\begin{tabular}{llll}
\hline & 1986 & 1996 & 2007 \\
\hline Entiende & $5305754(90,6 \%)$ & $5683237(95,0 \%)$ & $6610200(93,8 \%)$ \\
\hline Sabe hablar & $3760966(64,2 \%)$ & $4506512(75,3 \%)$ & $5331000(75,6 \%)$ \\
\hline Sabe leer & $3554442(60,7 \%)$ & $4330251(72,4 \%)$ & $5143100(73,0 \%)$ \\
\hline Sabe escribir & $1850967(31,6 \%)$ & $2743326(45,8 \%)$ & $3967500(56,3 \%)$ \\
\hline & & & \\
\hline Total & 5856433 & 5984334 & 7049900 \\
\hline (http://www20.gencat.cat/portal/site/Llengcat/menuitem)
\end{tabular}


De estos datos se deduce claramente que el nivel de compensión es elevado, que el número de aquellos que saben hablar y leer ha aumentado considerablemente, pero desde 1996 están casi paralizándose, mientras que la capacidad de escritura continúa aumentando. Sabemos que el estancamiento se debe en buena parte, a la inmigración, que muchas veces perjudica las lenguas autóctonas. Además, estas estadísticas no pueden enumerar todas las lenguas que entran en el campo de la comunicación: lo que sabemos gracias a encuestas cualitativas es que muchas lenguas del continente africano y algunas de Asia y de las Américas — no presentes o casi no presentes hace veinte años- han hecho su entrada en el país. Lo que no indica esta estadística es el uso real que los hablantes hacen de un idioma y otro. Existen muchas investigaciones que intentan dar una impresión del uso, pero las variables son tan complejas que no es fácil sacar conclusiones. Por un lado, se puede decir lo que Emili Boix dijo tan claramente en el título de su conocido libro Triar no és trahir, pero, por otro lado, es evidente que a lo largo del tiempo la capacidad de emplear un idioma disminuye, si disminuye mucho el uso. Si yo uso preferentemente un idioma, este idioma se fortifica en mi memoria y mis capacidades prácticas en él aumentan, mientras que paulatinamente disminuyen en las demás lenguas. Todas las encuestas muestran que las capacidades en catalán son superiores al uso de la lengua que se encuentra actualmente. Esto es, a largo plazo, un peligro para la lengua.

En lo que se refiere a Euskadi, las estadísticas en general no dan tantos detalles sino que diferencian a los que saben hablar de los que no saben hablar euskera. Se compara mucho menos entre las diferentes capacidades lingüísticas. Esto se debe, sin duda, al hecho de que la mayoría de los bilingües actuales adquirió su competencia a través de la enseñanza, en la cual los hablantes futuros aprenden no solo a entender y a hablar, sino que también los inicia en la lectura y la escritura. Los monolingües en vasco (euskaldunak), presentes en las primeras estadísticas de los años 80 y que representaban el 1\% de la población, han desaparecido. Hoy se cuenta únicamente con bilingües («la persona bilingüe habla bien tanto en euskera como en castellano») o hablantes, con bilingües pasivos («el bilingüe pasivo es capaz de entender bien el euskera, a pesar de no hablarlo bien») o usuarios, y con castellanohablantes monolingües (en el pasado este grupo se denominaba erdaldunak): 


\begin{tabular}{lll} 
& 1981 & 2006 \\
\hline Habitantes de más de 5 años & 1969038 & 2016257 \\
\hline Bilingües & $431129(21,9 \%)$ & $755640(37,5 \%)$ \\
\hline Bilingües pasivos & $240433(12,2 \%)$ & $349691(17,3 \%)$ \\
\hline Castellanohablantes monolingües & $1297476(65,9 \%)$ & $910926(45,2 \%)$ \\
\hline & (Gros, 2009, 111) & (Eusko Jaurlaritza, 2009)
\end{tabular}

En estas cifras se ve fácilmente el éxito de la oficialización y, sobre todo, el de la enseñanza del euskera (estos progresos se reconocen también en los anteriores Mapas sociolingüisticos del gobierno vasco). Por eso, el bilingüismo es más fuerte entre los jóvenes (76,7\% de entre 10 y 14 años según la misma fuente). Como las estadísticas de los otros territorios de Euskal Herria no son fácilmente comparables con estos datos, no se puede decir claramente cual es el conjunto de personas capaces de utilizar el euskera en cada una de las actividades comunicativas y, sobre todo, de participar en la vida cultural en esta lengua. En el caso de Euskadi, se ve otra vez que el uso real es inferior a las posibilidades teóricas: según la misma fuente, solo el 13\% (263 005 personas) utiliza en casa principalmente el euskera, otro 8,4\% (170 021) utiliza ambas lenguas. Pero el 78,5\% no utiliza el euskera en sus casas.

En general se dice que la competencia en Galicia es la más elevada de todas las comunidades autónomas. Desgraciadamente no dispongo de datos tan antiguos como los de las otras lenguas, pero las evoluciones también se pueden ver claramente. Las declaraciones se refieren a la competencia en gallego:

\begin{tabular}{lll} 
& $\mathbf{1 9 9 1}$ & $\mathbf{2 0 0 1}$ \\
\hline Entiende & $96,96 \%$ & $99,16 \%$ \\
\hline Sabe hablar & $91,39 \%$ & $91,04 \%$ \\
\hline Sabe leer & $49,30 \%$ & $68,65 \%$ \\
\hline Sabe escribir & $34,85 \%$ & $57,64 \%$ \\
\hline Total & \\
\hline (http://www.as-pg.com/php/upload/lin-1plandenormalizacionlinguagalega.pdf)
\end{tabular}

Estas cifras aparecen como muy satisfactorias, pero hay otras encuestas que conceden valores inferiores al gallego. No dispongo de las posibilidades para 
controlar estas indicaciones. Según la misma fuente, el uso del gallego sería bastante elevado; un 56,84\% (1 470 836) utilizaría siempre el gallego, un 30,29\% (783 780) lo utilizaría a veces y solo un $12,86 \%$ (332 791) no lo utilizaría nunca ${ }^{4}$. Lo que sé es que el prestigio y el valor comunicativo del gallego continúan siendo discutidos entre los mismos hablantes. Claro que el gallego es necesario en Galicia; pero como una parte importante de los habitantes entrevé la necesidad de una migración profesional, ya sea provisional o definitiva, el valor del castellano sigue siendo elevado. Por eso me pregunto si los valores indicados no son interpretaciones muy optimistas.

Queda claro que las tres regiones que acabo de presentar aquí son aquellas en las cuales las situaciones deberían ser las más positivas para las lenguas autóctonas. Es sabido que, en todas las otras, las políticas lingüísticas son menos decididas y que el papel oficial del castellano es mucho más importante que la lengua propia, sea cooficial o no.

Para terminar esta parte quisiera mostrarles otra presentación de los hechos, según las estadísticas oficiales (www.ine.es). Siguiendo esta fuente, que da porcentajes para seis comunidades autónomas, las situaciones son las siguientes:

\begin{tabular}{lll} 
Comunidad $^{5}$ & $\begin{array}{l}\text { Población que no entiende la } \\
\text { lengua propia de la comunidad } \\
\text { donde reside }\end{array}$ & $\begin{array}{l}\text { Población que domina la lengua } \\
\text { propia de la comunidad donde } \\
\text { reside }\end{array}$ \\
\hline Illes Balears & $12 \%$ & $37 \%$ \\
\hline Catalunya & $6 \%$ & $49 \%$ \\
\hline Com. Valenciana & $15 \%$ & $23 \%$ \\
\hline Galicia & $1 \%$ & $56 \%$ \\
\hline Navarra & $69 \%$ & $23 \%$ \\
\hline Euskadi & $43 \%$ & $46 \%$
\end{tabular}

\footnotetext{
${ }^{4}$ Durante el seminario algunos investigadores gallegos citaban encuestas recientes que indicaban solamente un uso de aproximadamente $30 \%$. Eso es muy preocupante.

${ }^{5}$ Las denominaciones y la ortografía de las comunidades son las que empleo en este artículo.
} 
En otras columnas se explica de qué manera la estadística llega a estos datos:

\begin{tabular}{lllllll} 
Comunidad & $\mathbf{1}$ & $\mathbf{2}$ & $\mathbf{3}$ & $\mathbf{4}$ & $\mathbf{5}$ & $\mathbf{6}$ \\
\hline Illes Balears & $12,4 \%$ & $21,0 \%$ & $6,4 \%$ & $7,7 \%$ & $15,9 \%$ & $36,7 \%$ \\
\hline Catalunya & $6,4 \%$ & $17,0 \%$ & $7,5 \%$ & $3,6 \%$ & $16,7 \%$ & $48,9 \%$ \\
\hline Com. Val. & $15,3 \%$ & $29,0 \%$ & $8,1 \%$ & $9,9 \%$ & $14,4 \%$ & $23,4 \%$ \\
\hline Galicia & $1,3 \%$ & $7,6 \%$ & $1,3 \%$ & $23,9 \%$ & $9,9 \%$ & $56,0 \%$ \\
\hline Navarra & $69,0 \%$ & $2,7 \%$ & $0,5 \%$ & $3,2 \%$ & $1,5 \%$ & $23,1 \%$ \\
\hline Euskadi & $43,4 \%$ & $3,8 \%$ & $0,9 \%$ & $4,6 \%$ & $1,4 \%$ & $45,8 \%$ \\
\hline
\end{tabular}

[Explicación: 1 = no lo entiende; 2 = solo lo entiende; 3 = solo lo entiende y lo sabe leer; 4 = solo lo entiende y lo sabe hablar; 5 = lo entiende y lo sabe hablar y leer, pero no escribir; 6 = lo entiende y lo sabe hablar, leer y escribir]

Las cifras indicadas en estas columnas son, en general, mucho más bajas que las que vimos anteriormente. Se define a una persona que domina la lengua propia únicamente como aquella que domina las cuatro destrezas lingüísticas. Todas las demás personas no se mencionan o se indican como no hablantes. Pero una lectura diferente de estos datos parece posible: si consideramos que, cuando uno sabe escribir, normalmente posee también las otras capacidades, y si el nivel mínimo es en primer lugar el de solo entender, seguido en segundo lugar por las capacidades de entender y hablar y, en tercer lugar de entender, hablar y leer, podemos establecer con los mismos datos una estadística que sugiere otra impresión:

\begin{tabular}{lllll} 
Comunidad & Entender & $\begin{array}{c}\text { Entender } \\
\text { y hablar }\end{array}$ & $\begin{array}{l}\text { Entender, } \\
\text { hablar y leer }\end{array}$ & Las cuatro \\
\hline Illes Balears & $87,6 \%$ & $60,3 \%$ & $52,6 \%$ & $36,7 \%$ \\
\hline Catalunya & $93,6 \%$ & $69,2 \%$ & $65,6 \%$ & $48,9 \%$ \\
\hline Com. Val. & $84,7 \%$ & $47,4 \%$ & $37,8 \%$ & $23,4 \%$ \\
\hline Galicia & $98,7 \%$ & $89,8 \%$ & $65,9 \%$ & $56,0 \%$ \\
\hline Navarra & $31,0 \%$ & $27,8 \%$ & $24,6 \%$ & $23,1 \%$ \\
\hline Euskadi & $56,6 \%$ & $51,8 \%$ & $47,2 \%$ & $45,8 \%$ \\
\hline
\end{tabular}

(Falta en esta presentación el grupo que «solo entiende y sabe leer», que oscila entre $0,5 \%$ en Navarra y $8,1 \%$ en la Comunidad Valenciana y que me suscita algunas dudas respecto a su practicabilidad) 
Si comparamos esta presentación con las que proponen las diversas instituciones de las autonomías, vemos que los datos son muy próximos a los que se indican allí. Lo que no sé es cuáles son las fuentes de estas cifras. Por eso no puedo afirmar que sean tan exactas como las proporcionadas por los interesados. De todas maneras, queda visible que la manera oficial de presentación da una impresión muy diferente, en la cual la presencia de las lenguas propias parece mucho más escasa que anteriormente. Honny soit qui mal y pense.

Todas estas columnas de cifras coinciden en unos elementos bastante claros: las lenguas propias han recuperado mucho después del fin de la dictadura, sobre todo en los ámbitos más culturales de la comunicación, quiero decir, en la lectura y la escritura. Solo en el caso del eusquera, también aumentó apreciablemente el mero conocimiento de la lengua, en parte debido a los bajos valores iniciales y en parte a la importancia simbólica que la lengua tiene para muchos vascos. Y eso se da sobre todo en las autonomías que han invertido mucho en la enseñanza y en los medios de comunicación (mass media). Veremos que, en los últimos años, hay nuevos factores que influyen sobre dichas evoluciones. Pero parece que el uso de las lenguas propias no sigue la misma evolución. En todos los casos que conocemos, el uso parece inferior a las competencias. Claro que eso se explica por muchas razones, unas muy conocidas, como el hecho de que el castellano sigue siendo la lengua oficial del Estado (art. 3.1 de la Constitución) y tomando de este estatus su prestigio (y una parte considerable de su valor comunicativo). No es necesario evocar estas razones nuevamente, que ya son conocidas. Lo que me parece útil es mencionar tres hechos que tienen un papel importante y que no estuvieron presentes hace treinta años atrás.

\section{NUEVAS VARIABLES DESDE LA PROCLAMACIÓN DE LA CONSTITUCIÓN DE 1978}

El éxito del castellano: El primero de estos elementos son los progresos mundiales de la lengua castellana en los últimos tres decenios. Permítanme un recuerdo personal: cuando empecé a estudiar el castellano, alrededor de 1960, en un curso en la universidad popular de mi ciudad, fue una decisión un tanto extraordinaria. En aquellos días se aprendían lenguas como el inglés, claro, el francés, 
el ruso e incluso el italiano, pero aprender el castellano era un tanto extravagante. Por consiguiente, había muchos cursos de inglés y de francés, algunos de ruso y de italiano, pero el castellano contaba únicamente con dos cursos, uno para principiantes y otro para avanzados — dictados por una señora de la edad de mi abuela-, a los que asistía muy poca gente (y siempre la misma). Cuando continué en la universidad, había un pequeño grupo que aprendía castellano, al lado de las masas que estudiaban el francés para dedicarse a la enseñanza de este idioma. Los estudios del castellano eran minoritarios porque el prestigio internacional de la lengua no era muy alto. Las interminables crisis del siglo XIX habían dejado una mala impresión, como fue el caso de la dictadura en España. América Latina estaba muy lejos y no parecía muy importante para estudiantes europeos; además los gobiernos allí no gozaban de una buena reputación. Tampoco se sabía mucho del mundo ibérico, y eso a pesar del turismo, que empezaba a tener un papel más importante en los años 60 .

Hoy vivimos en un mundo en el que el castellano es, después del inglés, la segunda lengua europea a nivel internacional. El francés y el ruso perdieron mucha importancia internacional en los últimos decenios (no hablo de lenguas no europeas), esto es un factor negativo, mientras que el castellano pudo mejorar considerablemente su prestigio y su valor comunicativo. En Europa, en las Américas $y$ en parte en otros continentes es una lengua que se emplea mucho, ya sea como lengua materna o como lengua escolar. Existen muchas razones para esto: el aumento absoluto y relativo de los hispanohablantes (en 1960 existían 100 millones de hispanohablantes en una tierra de 2,5 mil millones de seres humanos [un 4\%]; mientras que en 2007 se contaba con 358 millones de 6,7 mil millones de seres humanos [un 5,3\%], además 60 millones que lo dominan como segunda lengua, cf. Fischer 2008). Esto requiere más atención. Además el castellano «conquista» nuevos lugares, por ejemplo en EE. UU., donde anteriormente no gozaba de prestigio y en donde está a punto de conquistar cierto estatus oficial.

Más importantes me parecen otros factores: quizás, en primer lugar, el éxito de la democratización de España —éxito político, éxito económico, éxito social一, que hace que por primera vez se puede considerar a España como uno de los estados líderes de Europa. En este sentido, la integración en la Unión Europea en 1986 fue un paso importante. Este movimiento fue acompañado por evoluciones similares en algunos países de América Latina, como México (hoy el estado con el mayor núme- 
ro de hispanohablantes en el mundo). Estos éxitos confirman una cierta conciencia colectiva que ayuda a la imposición de normas cultas de la lengua en los respectivos estados, diferentes de las españolas (esto se produce sobre todo en Argentina y México, pero algunos otros estados siguen esta tendencia). Otro factor es la reputación mundial de la cultura latinoamericana, que empezó con el éxito de la literatura (sobre todo el realismo mágico) y que se popularizó con los bailes de América Latina. Me parece que este movimiento es muy importante porque confiere al castellano una impresión de «lengua de jóvenes» o de «lengua de futuro», que hace que muchos jóvenes quieran aprenderlo, y eso a pesar de las escasas posibilidades profesionales que ofrece. Y, también, hay que mencionar una política cultural y lingüística externa de España que multiplica los «Institutos Cervantes» en un momento en el que otros estados europeos como Francia cierran una parte de sus centros culturales. Está claro que esta evolución no se produce sin contradicciones y tensiones internas: los argentinos no están contentos con el hecho de que en Brasil se abran los «Institutos Cervantes» que enseñan una variedad europea del castellano, mientras que la norma argentina, mucho más útil —y más fácil— para los brasileños, no se emplea. Todo este conjunto de factores hace que el castellano se considere hoy como una de las lenguas más importantes y más utilizadas del mundo occidental. Toma actualmente un camino opuesto al que, de lengua dominante en Europa en el siglo XVI, le redujo a una posición muy limitada en el siglo XIX y la primera mitad del XX. Claro que esta evolución tan reciente le da una fuerza atractiva más grande al castellano que en el momento de la democratización, también dentro del Estado español.

La inmigración masiva: Otro elemento que puede perjudicar a las lenguas propias es la inmigración masiva reciente. Sabemos que hasta los años 60 España era un país de emigración: emigración definitiva en dirección al nuevo mundo, y emigración temporal en dirección a países europeos. El hecho de que esta emigración haya sido estacional, como la de los trabajadores agrícolas en Francia por ejemplo, o de más larga duración, como la de los trabajadores industriales en Alemania, Suiza y Bélgica, fue un factor importante en la evolución económica posterior en España. Hoy, la gran mayoría de los emigrados temporales ha vuelto a España (de los más de 500000 que vivían en Alemania, hace cuarenta años, quedan apenas 100000 hoy). Actualmente, asistimos a un movimiento contrario: España se ha convertido en un país de inmigración masiva. 
Este movimiento empezó aproximadamente con la implosión de los países socialistas y se intensificó con los movimientos recientes masivos de refugiados económicos y políticos que provienen de países europeos (Rumania, Moldavia, etc.), de América Latina (Ecuador, Colombia, etc.), pero sobre todo de países limítrofes africanos como Marruecos y de la zona del Sahel. Se calcula que hoy casi un $10 \%$ de la población de España son inmigrados (de primera o segunda generación), aunque es muy difícil obtener datos exactos. Esta inmigración plantea muchas veces un problema lingüístico para las autonomías: naturalmente, muchos inmigrados consideran de gran interés personal aprender rápidamente el castellano, pero no ocurre así con las lenguas cooficiales. Los que son oriundos de países multilingües aceptan en general bastante fácilmente las lenguas autonómicas, pero, curiosamente, los que se niegan con máxima intensidad a aprender estas lenguas son los inmigrados de procedencia latinoamericana, que piensan — por razones muy complejas - que el castellano tiene que bastar. De esta manera se explica la ligera reducción de los que entienden la lengua propia en los últimos diez años (esto es particularmente visible en Catalunya, uno de los destinos preferidos de inmigración). Claro que los gobiernos autonómicos han desarrollado políticas de integración y de aprendizaje de las lenguas propias para inmigrantes, ya que es necesario que estos, muchas veces enfrentados a problemas mucho más elementales, acepten estas iniciativas.

Pero no existe únicamente este tipo de inmigración de — digamos— «necesitados». Hay también una inmigración, menos importante numéricamente pero con un peso social considerable, de «ricos», fundamentalmente ingleses (275 000), alemanes (150 000) y, últimamente, rusos, sobre todo jubilados que quieren disfrutar de su vida bajo el sol mediterráneo. La mayoría de ellos no está dispuesta a integrarse de ninguna manera en la sociedad ambiental. Solo en el mejor de los casos dominan un mínimo de castellano y, en general, no quieren aprender otro idioma o amoldarse a otra cultura. Gran parte de ellos llegan con una especie de actitud colonial.

La presencia de muchos inmigrados estabiliza estructuralmente la situación de la lengua estatal a costa de las lenguas cooficiales.

El tercer elemento lo menciono solamente de memoria, porque para las políticas lingüísticas tiene hasta la fecha una importancia limitada: se trata de la entrada de España en la Unión Europea y en el Consejo de Europa, que obliga, por ejemplo, al respeto de ciertas normas jurídicas internacionales (en algunas partes de España toda- 
vía es un problema). Además, los últimos textos europeos han acentuado el respeto de los derechos humanos y permiten a los representantes de las autonomías encontrar otro nivel de interlocutores y neutralizar, de esta manera, al menos parcialmente, el centralismo estatal. Visto desde otra perspectiva, los organismos europeos (actualmente, sobre todo, el Consejo de Europa, pero también la Corte Europea de Derechos Humanos y la Corte de la Unión Europea) tienen potestad sobre algunos aspectos del problema y obligan a los gobiernos españoles sucesivos a modificar determinados elementos de sus políticas. Si los conflictos se intensifican - lo que actualmente parece posible- - puede ser que el papel de las instancias europeas sea mucho más importante en un futuro.

\section{LAS POLÍTICAS LINGÜÍSTICAS, SUS OBJETIVOS, SUS ALCANCES Y LÍMITES, LAS RESPECTIVAS LEYES}

Las primeras leyes intentaron dar contenido a la cooficialidad declarada por la Constitución y los estatutos. Era evidente que en los detalles tenían que diferenciarse porque la situación respectiva de las lenguas era diferente. En los casos en los que la normativización no estaba concluida (euskera y gallego), esta fijación fue la más urgente. Pero también en el caso del catalán era necesario un «aggiornamento» de la normativa; durante decenios las condiciones pésimas en el trabajo normativo impidieron reaccionar con la rapidez necesaria ante las evoluciones en el sector comunicativo: si en 1936 la radio era conocida y políticamente utilizada, nadie hablaba en aquel momento de televisión, algo muy banal en el momento de la transición. Las necesidades prácticas — construir un aparato de enseñanza que funcione- permitieron hacer progresos considerables en este sector, pero sabemos que algunas veces el precio a pagar fue muy alto: la no aceptación de la normativa fabriana por una parte considerable del público (no especializado) valenciano y la escisión lingüística parcial que resulta ${ }^{6}$, así como la falta de unanimidad respecto a la normativa en el dominio gallego continúan siendo algo preocupante.

\footnotetext{
${ }^{6}$ Antoni Maria Badia i Margarit empezó, hace unos veinte años, una conferencia de presentación de los problemas del catalán en Viena diciendo que anteriormente el catalán no gozaba de ninguna forma de reconocimiento del Estado mientras que en el momento contaba con tres formas diferentes de reconocimiento, lo que quería decir con tres normativas virtuales.
} 
Pero la normativización es la condición previa para la normalización, nada más y nada menos. Por eso era urgente implementar los pasos en dirección a una normalización eficaz. Quedaba claro que la importancia real de la propia lengua en el dominio tenía que dar los primeros pasos. Euskadi adopta, ya en 1982, una Ley básica de normalización del uso del Euskera, y Catalunya adopta en 1983 una Llei de normalització lingüistica a Catalunya, como lo hace Galicia, mientras que Navarra, las Islas Baleares y la Comunidad Valenciana esperan hasta 1986 para adoptar leyes comparables, pero mucho más restrictivas, para la lengua propia respectiva. Las diferencias entre los títulos ya muestran las diferencias que las leyes tenían que respetar: mientras que en Catalunya una gran mayoría de la población entendía un idioma normativizado, que poseía los instrumentos más importantes para la normalización, y era solamente analfabeta en esta lengua, en Euskadi era necesario terminar el trabajo de normativización y elaborar los instrumentos básicos para empezar, por ejemplo, una enseñanza de masas (por cierto, las ikastolak ya habían preparado el terreno, pero quedaba mucho por hacer). Además, en Euskadi el euskera era la lengua de una minoría, lingüísticamente muy distante de la lengua dominante, mientras que la intercomprensión entre lenguas iberorrománicas es bastante fácil. La política en Euskadi tenía que asegurarle un espacio en la vida pública al euskera, mientras que en Catalunya el catalán aspiró, ya en los comienzos, a ocupar el lugar como primera lengua pública. Por eso se veía rápidamente que la ley de normalización de 1983 no era suficiente; consecuentemente, el Parlamento catalán aprobó, después de una muy larga preparación, en 1997-98, una nueva Llei de política lingüistica. Esta nueva ley fue el primer paso hacia el nuevo estatuto de autonomía de 2006. Pero sus razones de existencia no son solamente positivas: hacen ver que la primera ley no era suficiente para conseguir que el catalán ocupase el lugar deseado. Un aparato prescriptivo más detallado debía cerrar las puertas por las cuales el castellano entraba en sectores claves de la vida cotidiana y algunas veces impedía que el catalán progresase. En ese momento, se podía observar que la unanimidad con la cual las primeras leyes fueron aprobadas en los primeros años de los 80 no continuaba existiendo: el PP, nacionalista español, se opuso porque pensaba que la normalización tenía que limitarse a ciertos aspectos, mientras que ERC, nacionalista catalán, pensaba que esta ley no cumplía con todos los aspectos de la normalización. El PP decidió oponerse por vía jurídica y, efectivamen- 
te, algunos párrafos de la ley fueron abolidos por la justicia (lo había hecho ya en 1983 el PSOE para combatir la ley anterior). Desde este instante, se observa una ruptura entre partidos nacionalistas españoles, que quieren limitar los derechos autonómicos, y partidos periféricos, que intentan aseguralos y, alguna vez, ampliarlos.

Claramente, esta contradicción se actualiza, hasta la fecha, únicamente para los poderes regionales que quieren cruzar la línea invisible de compromiso que trazó la Constitución de 1978: esta línea concede a las lenguas propias más de un mero derecho minoritario pero menos que una cooficialidad que podríamos llamar simétrica. En muchos casos, los gobiernos autonómicos no se atreven a cruzar esta línea: esto es evidente en todos los casos en los cuales las lenguas autóctonas gozan del reconocimiento mínimo según el art. 3.3 de la Constitución, pero también en algunos casos de cooficialidad, por ejemplo en la Comunidad Valenciana, en Navarra, durante mucho tiempo en la Islas Baleares y en Galicia. Los gobiernos baleares de 1999-2003 y desde 2007 acentúan su política en favor del catalán, pero, al revés, el gobierno de PP electo en 2003, anula algunas disposiciones en favor de la lengua propia del gobierno anterior, mostrando de esta manera que como partido nacionalista español no estaba dispuesto a aceptar medidas demasiado pro-catalanas. Si el gobierno PSOE / BNG en Galicia de 2005-2009 acentuó un poco la política pro-gallega — no demasiado ya que el primer partido gubernamental fue un partido español—, parece que la mayoría actual no continuará en esta misma dirección, sino que disminuirá severamente las posibilidades del gallego, lo que ya hace nacer movimientos reivindicativos nuevos en favor del gallego (2010). Por su parte, la opinión pública en favor del gallego no es tan fuerte ni tan unida para poder imponer — por el momento— una política más decidida al poder autonómico (lo que se produce regularmente tanto en Catalunya como en Euskadi). Los poderes autónomos de la Comunidad Valenciana, desde la primera victoria del PP en 1994, han acentuado la política lingüística anti-catalana, insistiendo en las diferencias entre catalán y valenciano y creando, de esta manera, una tensión interna, cuyos términos hacen reír al mundo extranjero, pero que impide eficazmente una política real de cooficialidad. El catalán valenciano está lejos de jugar el papel que podría y que, según la ley, debería jugar. En la situación de asimetría en la que se encuentra en este momento, existen naturalmente consecuencias para el uso de la lengua. 
Quedan por mencionar, entonces, los dos casos de Catalunya y Euskadi. Ambas autonomías han intentado o intentan cruzar dicha línea, ya sea con la clara voluntad de someter la pertenencia de la región al Estado español a un plebiscito (Euskadi) y de entrar de esta manera en contradicción abierta con la Constitución Española, o bien con un trabajo que a menudo exige algunos pasos suplementarios para aproximar al primer lugar la lengua autóctona y para el reconocimiento del grupo autóctono como nación y — hasta la fecha (2010) — sin hablar (mucho) de independencia política (Catalunya). En estas luchas de nacionalismos opuestos, parece que nos encontramos en un punto «muerto» actualmente. En Euskadi, por un conjunto de medidas jurídicas (interdicción de diversos partidos abertzales de izquierda) y políticas (formación de un gobierno socialista sin apoyo de partidos abertzales, pero sostenido por el PP), el problema actualmente parece neutralizado. El gobierno actual le da una importancia mucho menor a la política lingüística y cultural que sus antecesores, y espera un éxito más duradero insistiendo sobre una política económica más activa. La crisis económica actual no permite decidir si esta estrategia es prometedora. Lo que hay que constatar es que hasta ahora parece haber menos resistencia de lo previsto. Pero el problema no está resuelto, simplemente ha sido aplazado.

Para Catalunya el estatuto de 2006 es una victoria relativamente parcial. Parcial porque algunos puntos simbólicamente importantes fueron anulados por las Cortes españolas y porque creo que este estatuto muestra que la política de promover el catalán realmente al puesto de primera lengua de Catalunya se ha logrado solamente en parte y que el futuro parece preocupante. En este contexto, la sentencia del Tribunal Constitucional de junio de 2010 y las reacciones que provocó, aunque sea quizás menos restrictiva de lo que parezca, puede significar - para un futuro todavía no previsible - un punto de ruptura: muchos catalanes ahora tienen la impresión de que con Madrid nunca podrán lograr sus aspiraciones, lo que los obliga a pensar en soluciones alternativas, es decir, cómo llegar a soluciones sin Madrid. En el marco de la constitución actual esto no parece posible. Por ejemplo, una solución como la de la provincia de Québec, en Canadá, monolingüe en francés en un estado bilingüe canadiense, no es posible según la constitución actual.

La solución más apropiada sería, sin duda, la de una reforma constitucional que se desentienda de algunos de los compromisos de la transición para llegar a 
una simetría mayor de las diferentes lenguas y culturas y que, así, facilite a los pueblos periféricos la pertenencia al Estado español, pertenencia que en el momento de la construcción europea pierde importancia y de esta manera tendría que hacerse cada vez más soportable. Por otro lado, si la España centralista no está dispuesta a concesiones suplementarias, es posible que las fuerzas centrífugas algún día sean más fuertes que las otras y que Europa prefiera una separación a un conflicto sin fin. Actualmente, no hay consenso político suficiente para lograr esta revisión de la constitución. Por eso, las contradicciones internas continuarán y las mentes van a envenenarse mutuamente por falta de la posibilidad de lograr compromisos aceptables por todos (compromisos, añado, que desde un punto de vista externo, no parecen imposibles de realizar). Si los nacionalistas españoles no están dispuestos a gestos y cambios de actitudes, no es imposible que el precio a pagar un día sea muy elevado. 


\section{BIBLIOGRAFÍA}

BAUER, O.: Die Nationalitätenfrage und die Sozialdemokratie, Wien, Verlag der Volksbuchhandlung, 1907 [1924²].

BeIn, R.: «Die Wechselwirkung Prestige / Gebrauchswert des Französischen: früher erste, heute dritte Fremdsprache in Argentinien», en J. BORN (Hg.), Mehrsprachigkeit in der Romania, Französisch im Kontakt und in der Konkurrenz zu anderen Sprachen, Wien, Praesens, 2001, pp. 82-91.

BoIX [I FUSTER], E.: Triar no és trahir. Identitat i llengua en els joves de Barcelona, Barcelona, Ed. 62, 1993. Doppelbauer, M.: «La Constitución y las lenguas españolas», en M. Doppelbauer / P. CichON (eds.), La España multilingüe. Lenguas y políticas lingüisticas de España, Wien, Praesens, 2008, pp. 21-30.

Eusko Jaurlaritza / Gobierno Vasco: IV Mapa Sociolingüistico, Vitoria-Gasteiz, Kultura Saila / Departamento de Cultura, 2009.

FERrANDO, A. y M. NicOlÁs (a cargo de): La configuració social de la norma lingüistica a l'Europa llatina, Alacant, Institut Interuniversitari de Filologia Valenciana, 2006.

FERRER, M.: «Aspectos geográficos», en F. FERNÁNDEZ RodríGUeZ (coord.), La España de las autonomías, Madrid, Instituto de Estudios de Administración Local, 1985, pp. 1-67.

FISCHER WELTALMANACH 1977: Frankfurt am Main, Fischer Taschenbuch Verlag, 1976.

FISCHER WELTALMANACH 2008: Frankfurt am Main, Fischer Taschenbuch Verlag, 2007.

FISCHER WELTALMANACH 2010: Frankfurt am Main, Fischer Taschenbuch Verlag, 2009.

Generalitat de Catalunya: Estatut d'autonomia de Catalunya 2006, Barcelona, Generalitat, 2006.

Gros i Lladós, M.: El euskera en la Comunidad Autónoma Vasca (2009). Una apuesta por la diversidad lingüistica, Bilbo / Bilbao, Euskaltzainida, 2009.

Hroch, M.: Die Vorkämpfer der nationalen Bewegung bei den kleinen Völkern Europas, Prag, Karls-Universität, 1968.

- Das Europa der Nationen. Die moderne Nationsbildung im europäischen Vergleich, Göttingen, Vandenhoeck \& Ruprecht, 2005.

INE: http: //www.ine.es

KremnitZ, G.: Gesellschaftliche Mehrsprachigkeit. Institutionelle, gesellschaftliche und individuelle Aspekte, Wien, Braumüller, 1990 [1994²].

— «Le concept du "conflit linguistique" aujourd'hui. Essai d'une mise à jour. Avec une annexe: Quelques remarques sur le terme de "valeur communicative" des langues", Lengas (Montpellier), n. ${ }^{\circ} 54$ (2003), pp. 7-22.

- «Sur la délimitation et l'individuation des langues. Avec des exemples pris principalement dans le domaine roman", Estudis Romànics (Barcelona), XXX, (2008), pp. 7-38.

— «Les auteurs littéraires et leurs choix linguistiques: quelques observations sur l'évolution d'une relation compliquée», en F. BRUGNOLO (a cargo de), Scrittori stranieri in lingua italiana dal Cinquecento ad oggi, Padova, Unipress, 2009 [2010], pp. 3-13.

MAAS, U.: «Politique de la langue. Concepts de base pour la linguistique politique», Lengas, n. 9 (1981), pp. 9-38. 
Monteagudo, X. H.: «Precondicións sociais do proceso de conformación da norma do galego contemporáneo», en A. FerRANDO y M. NiCOLÁs (a cargo de), op. cit., 2006, pp. 301-341.

QUerol I PUIG, E.: El coneixement del català 2001. Mapa sociolingüistic de Catalunya. Anàlisi sociolingüistica del cens de població de 2001, Barcelona, Secretaría de Política Lingüística, 2006.

STALIN, J. W.: Marxismus und nationale Frage, Wien, 1913.

Tierno Galván, E. y A. Rovira: La España autonómica, Barcelona, Bruguera, 1985. 